whether the covariates (age, sex, BMI, KL score, number of AHMs) modified the association of SBP, DBP and PP with cartilage T2. RESULTS/ANTICIPATED RESULTS: The average age of all study participants was 58.8 years $(\mathrm{SD} \pm 8.6)$ with a higher proportion of men (59.4\%), average body mass index (BMI) was 28.3 ( $\mathrm{SD} \pm 4.5$ ), average SBP was $122.4(\mathrm{SD} \pm 15.4) \mathrm{mmHg}$, average DBP was 75.5 $(\mathrm{SD} \pm 9.6) \mathrm{mmHg}$ and 469 (38.1\%) study participants were taking at least one AHM. Higher baseline DBP was significantly associated with a faster increase in global T2 $(0.22[0.10,0.35], \mathrm{P}<0.001)$, global deep layer T2 $(0.20[0.03,0.36], \mathrm{P}<0.022)$ and global superficial layer T2 $(0.39$ [0.20,0.58], $\mathrm{P}<0.001)$. These associations were significant in both unadjusted and the models adjusted for age, sex, $\mathrm{BMI}$ and KL score. No significant associations were found between SBP or PP and cartilage T2 and no significant interactions were found between SBP, DBP, PP and the covariates. DISCUSSION/ SIGNIFICANCE OF IMPACT: Higher baseline DBP was associated with a faster increase in knee cartilage T2, suggesting accelerated cartilage degeneration. This association was stronger for the superficial layer of knee cartilage T2 compared to the deep layer. Although further basic mechanistic studies are needed to elucidate the underlying pathophysiology of this relationship, these results suggest lowering DBP may influence knee OA.

3206

Associations between prenatal maternal stress due to a natural disaster and effortful control in early childhood Nayra del Carmen Rodriguez-Soto ${ }^{1}$ and Karen G Martinez, MD, MSc ${ }^{1}$ University of Puerto Rico-Medical Sciences Campus

OBJECTIVES/SPECIFIC AIMS: For this reason, our overall objectives are to determine (i) whether natural disaster-related PNMS alters infants' EC at two years of age, and (ii) if the timing of exposure moderates its effects on toddlers EC. METHODS/STUDY POPULATION: We propose a longitudinal study with 50 mothertoddler dyads. Natural disaster-related PNMS would be measured at 12-18 and 24-30 months of age and will include: objective exposure and maternal distress. EC will be measured with a questionnaire and a Laboratory Temperament Assessment Battery at two years of age. To accomplish our objectives, we will conduct regression and moderation analyses. RESULTS/ANTICIPATED RESULTS: We anticipate that children exposed to Hurricane-related PNMS would present low EC levels compared to those with low prenatal exposure. DISCUSSION/SIGNIFICANCE OF IMPACT: These results are expected to provide evidence for further promoting early intervention and ameliorating adverse effects of PNMS on child outcomes.

3200

\section{Balancing Cost and Quality of Care in the US and Denmark: Lessons for Nations Transitioning from Volume-Based to Value-Based Care \\ Negin Fouladi ${ }^{1}$ and Margit Malmmose, PhD, MSC \\ ${ }^{1}$ University of Maryland School of Public Health}

OBJECTIVES/SPECIFIC AIMS: Promote knowledge translation and evidence-informed decision-making by assessing barriers and facilitators to balancing cost and quality of care within the US state of Maryland and nation of Denmark. METHODS/STUDY POPULATION: Open-ended and semi-structured key-informant interviews were conducted in 2016 and 2017 among high level decision-makers in Maryland $(\mathrm{N}=21)$ and the Danish $(\mathrm{N}=17)$ healthcare systems, including hospital, local, regional, and cross-organizational administrators and elected officials. The interviews consisted of questions related to: (1) currently practiced and preferred approaches to resource allocation and development and use of quality performance measures, and (2) preferred sources, formats/styles, modes of information, and decision-making strategies based on a shift from volume to quality-driven care. RESULTS/ANTICIPATED RESULTS: Decision-makers in Maryland expressed the need for collaboration in a changing environment, yet increasingly rely on cost and quality outcomes data to drive decisions and note the struggle to identify credible and useful information. Maryland decision-makers also face challenges in regulating utilization and costs without mandated participation of physician practices within the global budget cap model, which is perceived to be a primary driver of healthcare utilization in the hospital sector. Similarly, decision-makers in Denmark conveyed the importance of quantitative data to aid decisions, however, stress collaboration and dialogue as driving factors and important sources of information. Danish decision-makers also express challenges to wide-spread adoption of a quality-driven approach due to unsustained quality assurance regulatory bodies. DISCUSSION/SIGNIFICANCE OF IMPACT: The findings suggest implementation of value-based healthcare is highly driven and influenced by availability of credible data, which may significantly impact development of policies and innovative cost control strategies, and regulatory oversight to promote adoption of quality measures in decision-making. Furthermore, collaboration within and across healthcare organizations remains a key component to health system improvement as it fosters dialogue and sharing of best practices among stakeholders.

3022

\section{Barriers to Accessing Follow-up Care and Changes in Medical Needs after Childhood Injury}

Teresa Maria Bell ${ }^{1}$, Ashley N Vetor, Dennis P Watson, Christopher A Harle and Aaron E Carroll

${ }^{1}$ Indiana University School of Medicine

OBJECTIVES/SPECIFIC AIMS: The objective of this study was to prospectively assess caregiver-perceived barriers to accessing post-acute care for their injured child and determine if caregivers report ongoing, unmet health needs for their children after trauma. METHODS/STUDY POPULATION: This was a prospective cohort study that followed 50 participants for 6 months and administered surveys to parents of children who are admitted to a pediatric level 1 trauma center for injury. Surveys were given bi-weekly regarding care children received after hospital discharge. At 3 months, parents were surveyed over the phone on whether they were able to access all needed health services and if there were any perceived barriers to obtaining or providing at-home care. At 6 months, parents were given the Child \& Family Follow-up Survey to assess ongoing physical, mental, social, and scholastic needs. Free responses and transcribed interviews were analyzed using thematic content analysis and frequencies are reported for discrete data. RESULTS/ ANTICIPATED RESULTS: Out of 50 families recruited, 47 completed follow-up assessments. At 3 months, common themes regarding challenges after hospital discharge included difficulty scheduling specialist care; uncertainty in managing their child's pain; transitioning home without enough knowledge to meet their child's medical 
needs; lack of communication between multiple providers; distress at having providers release children to full activities before caregivers were comfortable. At 6 months, approximately 24\% of parents reported children had ongoing cognitive limitations, 29\% reported emotional problems, $19 \%$ reported physical limitations, $33.3 \%$ reported difficulty in school, and 15\% reported play/social difficulties. DISCUSSION/SIGNIFICANCE OF IMPACT: Evidence suggests families face significant barriers in accessing follow-up care, despite nearly universal health insurance coverage for children. Further, a large percentage of parents report ongoing health needs, despite the majority of the cohort having only mild or moderate severity injuries. Making follow-up care more patient-centered for families of traumatically injured children may improve compliance with medical regiments and reduce the likelihood of future disability. Examples of this may be coordinating care among multiple specialty providers, so that patients with multiple injuries can schedule multiple follow-up appointments on the same day. Additionally, more caregiver education on administering pain medication, caring for wounds, and safe practices for returning to full activities would be beneficial for families.

\section{Cell Proliferation and Differentiation in 3D printed Polycarbonate Urethane Porous Scaffolds}

Bijan Abar ${ }^{1}$, Alejandro Aalleja, Cambre Kelly, Natalia Von Windheim, Jennifer West and Kenneth Gall

${ }^{1}$ Duke University

OBJECTIVES/SPECIFIC AIMS: The aim of this study is to understand how porosity and collagen filling impact cell proliferation and differentiation in 3D printed scaffolds. METHODS/STUDY POPULATION: 3 groups of scaffolds will be 3D printed using FDM: solid scaffold, porous scaffold and porous scaffold with collagen gel ( $\mathrm{n}=10$ for each group) Internal geometries and surface structure will be analyzed using micro CT and Scanning Electron Mi RESULTS/ ANTICIPATED RESULTS: We hypothesize that porosity and collagen filler will increase signal from Picogreen assay and ALP assay when normalized to scaffold surface area, indicating enhanced cell proliferation and differentiation. DISCUSSION/SIGNIFICANCE OF IMPACT: 3D printing PCU is a relatively new technique with very little published in the literature. Previous work has focused on the mechanical properties and not the biological response to the polymer. Understanding how to optimize cellular proliferation and differentiation can lead to the development of better implants that will integrate into the host's structure and facilitate tissue regeneration.

\section{3}

\section{Comparative Effectiveness of Levetiracetam versus Phenobarbital for Infants Aged One Month to One Year, using US Medicaid Claims \\ Michelle Yun ${ }^{1}$, Manyao Zhang ${ }^{1}$, Alan Wu ${ }^{1}$, Natasha Basma ${ }^{1}$ and Zachary Grinspan ${ }^{1}$ \\ ${ }^{1}$ Weill Cornell}

OBJECTIVES/SPECIFIC AIMS: Using national Medicaid claims, this retrospective cohort study aims to compare the outcomes of levetiracetam (LEV) versus phenobarbital (PHB) as initial monotherapy in infants with epilepsy aged one month to one year. We primarily analyzed health services outcomes, as follows: (1) Emergency Department (ED) visits: proportion of those with at least $1 \mathrm{ED}$ visit, time to first $\mathrm{ED}$ visit, total number of $\mathrm{ED}$ visits, proportion leading to inpatient admission (2) Inpatient Admissions: proportion of those with at least 1 admission, time to first admission, total number of inpatient admissions, total length of stay (3) Treatment discontinuation: proportion of those who discontinued medication, time to discontinuation, proportion of those with prescription of second antiepileptic drug (AED) (4) Cost: total cost at 1 year from first prescription. METHODS/STUDY POPULATION: The project is a retrospective cohort study utilizing Medicaid claims from 2009-2012 from all states and DC. Inclusion criteria were: (1) Epilepsy diagnosis (ICD9 345.x) at age 1 month to 1 year (2) 1 year of continuous Medicaid enrollment Exclusion criteria were: (1) Neither LEV nor PHB monotherapy as initial treatment (2) Neither LEV nor PHB prescription within 45 days of epilepsy diagnosis (3) Less than 1 year of follow-up after first LEV or PHB prescription (4) Infantile Spasms diagnosis (5) Brain Surgery (6) Death within 1 year of follow-up Using $\mathrm{R}$ for statistical analysis, we analyzed outcomes including ED visits, inpatient admissions, treatment discontinuation, and total cost. In this abstract, we present our preliminary bivariate analysis. RESULTS/ANTICIPATED RESULTS: Demographics and Etiology: Compared to infants prescribed PHB $(n=1954)$, infants prescribed LEV $(\mathrm{n}=1248)$ were older (median 6 months [IQR 3-9] vs 3[2-6]; $\mathrm{p}<0.001)$, more likely to be white $(44.2 \%$ vs $38.9 \%$; $\mathrm{p}<0.05)$ and not Hispanic $(63.5 \%$ vs $58.9 \%$; $\mathrm{p}<0.05)$. There were also important differences in epilepsy etiologies $(\mathrm{p}<0.05)$. For example, infants prescribed LEV were more likely to have a diagnosis of tuberous sclerosis ( $1 \%$ vs $0.15 \%$ ) or traumatic brain injury ( $12.8 \%$ vs $0.56 \%)$. Health Services Outcomes: After 1 year, infants prescribed LEV had more ED visits $(2[0-4]$ vs $1[0-3] ; p<0.001)$ but shorter inpatient length of stay when admitted (3 days [2-5] vs 3 [2-6]; $\mathrm{p}<0.001)$. They were less likely to discontinue the medication $(46.6 \%$ vs $64.3 \% ; \mathrm{p}<0.001)$ but more likely to have a second AED prescription $(53.3 \%$ vs $43.4 \%$; $p<0.001)$. Other outcomes, including total cost, were similar. DISCUSSION/SIGNIFICANCE OF IMPACT: This preliminary analysis suggests that the healthcare trajectory of infants treated with LEV and PHB differ in complex ways. In ongoing work, we are conducting a multivariable comparative effectiveness analysis of LEV versus $\mathrm{PHB}$ using propensity score weighting to account for observable selection bias and multiple imputation to account for missing data.

3245

Comparison of Health Outcomes in Adolescent and Young Adult (AYA) Oncology Patients Treated at a Pediatric versus Adult Institute

Amy Moskop ${ }^{1}$, Julie Panepinto and Sadie Dobrozsi

${ }^{1}$ Medical College of Wisconsin

OBJECTIVES/SPECIFIC AIMS: Objectives: To examine the differences in health outcomes of adolescents and young adults treated for malignancy at a pediatric cancer center compared to an adult cancer center. To determine the differences in receipt of supportive care for adolescents and young adults with malignancy who are treated at a pediatric cancer center compared to an adult cancer center. METHODS/STUDY POPULATION: Methods: This will be a retrospective cohort study of patients from the ages of 15 to 